\title{
La adquisición
}

del sistema verbal

del español. Datos

empíricos del proceso

de aprendizaje del

español como lengua

extranjera.

Maria Cecilia Ainciburu (ed.).

Frankfurt: Peter Lans, a. M., 2017.

\section{Graciela Vázquez}

Recebido em: 31 de dezembro de 2017

É doutora em Linguística Aplicada e professora na Freie Universität de Berlin. Suas áreas de pesquisa são o discurso acadêmico, a interculturalidade e a didática do espanhol como língua estrangeira. Coordena diversos projetos europeus e é autora de numerosos publicações e de materiais de ensino.

Contato: sraciela.vazquez@fu-berlin. de 
El aprendizaje del castellano como lengua extranjera (ELE) carece todavía de una investigación empírica que fundamente de manera coherente y sinergética los enfoques didácticos del siglo XXI. La mayor parte de los estudios giran en torno a los procesos de enseñanza y adquisición del inglés e incluso los documentos programáticos más influyentes son de origen anglosajón y responden a necesidades no compartidas en todas las áreas de aplicación. Si bien llegamos al siglo XXI con un bagaje teórico sumamente rico si comparamos el estado actual con la situación de ELE hace medio siglo, no cabe duda de que la obra que reseñamos llena un vacío en un área clave: el sistema verbal. Podríamos plantearnos por qué concentrarnos en un tema gramatical en la era de las competencias, los enfoques interintraculturales y el aprendizaje estratégico. Dicho de otro modo: ¿En qué medida esta publicación contribuye a un orden lógico en la presentación y progresión de muestras de lengua y sus variedades? ¿Se aclaran aspectos del sistema verbal desde la perspectiva de una gramática pedagógica? ¿Dilucida el concepto de competencia gramatical en hablantes de lenguas extranjeras? ¿Intenta resolver la cuestión de cuánta gramática es necesaria para funcionar comunicativamente? ¿Hasta qué punto la interlengua o interlenguas como sistemas permeables de acercamiento al perfil del hablante nativo (hoy por hoy tendiente a desaparecer en favor de hablantes interculturales) puede variar en función no solo de la L1 sino de otras lenguas aprendidas? Los trabajos aquí presentados, de un modo u otro, ayudan a reflexionar sobre casi todas estas cuestiones. De no ser así, estaríamos frente a una serie de estudios sobre adquisición (un fin en sí mismo, por otro lado) pero no contribuirían a cerrar la brecha entre la investigación básica y aplicada, es decir, la investigación y el aprendizaje en contextos formales. Desde un punto de vista meramente práctico, estos trabajos facilitan el acceso a 
LA ADQUISICION DEL SISTEMA VERBAL DEL ESPAŃOL. DATOS EMPÍRICOS DEL PROCESO DE APRENDIZAJE DEL ESPAŃOL COMO LENGUA EXTRANJERA.

Maria Cecilia Ainciburu (ed.). Frankfurt: Peter Lang, a. M., 20 i 7.

Graciela VÁzQuez

resultados de otro modo dispersos en tesis doctorales (unidas aquí por el sistema verbal como denominador común)y se presentan enmarcados a través de un prólogo que, a grandes rasgos, describe brevemente las líneas generales (María Cecilia Ainciburu, editora), seguido de un primer capítulo que subraya la importancia de la perspectiva multifactorial tanto en el nivel del sentido como en el del significado (María Luisa Regueiro), para terminar con un análisis desde la perspectiva psicolingüística y el rumbo de futuras investigaciones (Marta Baralo Otonello). Los ocho artículos conforman una suerte de poliedro, con cada faceta dedicada a un tema individual dentro de una línea general. A continuación, paso a la presentación de cada capítulo, sin perder de vista quién puede beneficiarse de su lectura y en qué consiste la aportación concreta.

\section{Capítulo 1: Maria luisa Regueiro "El sistema verbal como eje de la reflexión semántica"}

Excelente acercamiento temático y aguda selección de la bibliografía referente a las posiciones intervencionistas y no intervencionistas, como así también una revisión acertada de las gramáticas existen tanto normativas como pedagógicas. La autora pone en tela de juicio la (in-) utilidad de las nomenclaturas utilizadas en los libros de texto y propone una clasificación de funciones semánticas. Sin embargo, cabría plantearse si las etiquetas propuestas evocan las mismas imágenes en diferentes culturas y si los significados están sujetos a las mismas limitaciones. Una revisión de conceptos tales como tiempo, aspecto, modo y diátesis media pueden enriquecer la competencia gramatical de quienes enseñan y facilitan explicaciones

a preguntas habituales relacionadas con errores comunes y frecuentes. 
Es posible visualizar una serie de actividades basadas en la tipología que propone la autora, aunque el bagaje léxico que estas requieren pareciera ser más asequible a niveles a partir de B1. Esto nos lleva a reflexionar sobre la posibilidad de diseñar gramáticas más que por contenidos y funciones (Plan Curricular del Instuto Cervantes, por ejemplo) por sentidos y significados. El capítulo ofrece una excelente revisión teórica sin perder la perspectiva aplicada, y resulta de gran interés para el futuro profesorado.

\section{Capítulo 2: IgnaCio Arroyo HernÁndez "LA adouIIICIÓN de CONSTRUCCIONES EXISTENCIALES Y}

\section{LOCATIVAS CON 'HABER' Y 'ESTAR'"}

Tras subrayar la importancia de las construcciones existenciales y locativas en la comunicación, el autor señala las limitaciones que detentan las explicaciones de los libros de texto de uso corriente y propone un modelo alternativo basado en la experimentación que permita una solución a nivel teórico, pero que simultáneamente satisfaga las exigencias de una gramática pedagógica sobre la cual construir actividades de clase. La dificultad que presentan estas estructuras - que pueden considerarse opacas - se explica a través de tres factores que dan cuenta de su falta de transparencia y que son comparables con diferentes tipos de interferencia, lo cual a su vez es indicador del potencial de dificultad. Tomar conciencia de los factores que permiten catalogar ciertas estructuras como opacas es una manera altamente efectiva de explicar la presencia, permanencia y variabilidad de algunas construcciones en la interlengua, lo cual permitiría monitorear la producción. El capítulo presenta una propuesta didáctica basada en lo que se conoce como "atención a la forma y procesamiento del input" como 
LA ADQUISICION DEL SISTEMA VERBAL DEL ESPAŃOL. DATOS EMPÍRICOS DEL PROCESO DE APRENDIZAJE DEL ESPAŃOL COMO LENGUA

alternativa a otras formas de instrucción más tradicionales, y a pesar de que el grupo meta es ítalo parlante, es posible extender los resultados a hablantes de otras lenguas. Un valor agregado del trabajo radica en la autocrítica desplegada en relación a la metodología de investigación empleada, lo cual puede contribuir de manera ejemplar al entrenamiento de investigadoras o investigadores en ciernes.

\section{Capítulo 3: Maria José Barrios Salvador "La expresión de la probabillidad en el nivel}

\section{B2. DIAGNÓstICO DE DIFICULTADES"}

La autora de este artículo aborda el tema de la dificultad que plantea para hablantes anglófonos de nivel B2 la elección modal que requiere una serie de operadores de probabilidad con los cuales la selección modal puede ser obligatoria o donde se observa una alternancia. El estudio demuestra que la atención que se dedica al valor léxico de dichos operadores neutraliza la selección modal. Se sopesan con mucho criterio los resultados obtenidos marcando ventajas y desventajas en la metodología de investigación y planteando sugerencias para mejorar la implementación en futuras replicaciones del experimento. A pesar de que el tema es importante, el capítulo presenta ciertas imprecisiones: la diferencia entre niveles no reside en el conocimiento de ciertos aspectos gramaticales; ceñirse al Plan Curricular del Instituto Cervantes podría ser un punto de apoyo pero no es necesariamente un documento ampliamente aplicado al desarrollo de materiales; hubiera sido recomendable la comparación con otros trabajos. También habría que tener en cuenta que si se compara el estudio con otros corpora (CAES, por ejemplo), podríamos encontrarnos con otras estructuras que no encajan 
en los niveles del Plan Curricular y en producciones del estudiantado que responden a otras variantes del castellano y no necesariamente a la gramática normativa . De todos modos, la selección modal representa uno de los mitos en cuanto al concepto de dificultad y el capitulo constituye un buen ejemplo de esta problemática.

\section{Capítulo 4: InÉS Fessi "Adouisición y transferencia de tiempo y aspecto en el español como} L3"

Este estudio introduce una perspectiva muy actual en el panorama del aprendizaje de lenguas: se refiere la a importancia que puede detentar la transferencia positiva o negativa de otras lenguas aprendidas, concretamente en lo que respecta a la temporalidad y al aspecto. Tras una introducción descriptiva de la similitud del sistema verbal en castellano y francés y de las diferencias de estas lenguas con respecto al árabe, seguida por una excelente relación bibliográfica de las publicaciones más recientes, la autora presenta los resultados de su propia investigación, a través de la cual demuestra que la tendencia a transferir de una L2 no es automática pesar de las similitudes tipológicas. Esto plantea un desafío metodológico interesante. Por un lado, en el plano comunicativo, es posible sobrevivir con una sola forma del pasado en alternancia con el imperfecto que carece del rasgo + tiempo y aporta modalidad. Por otro lado, si bien todo aprendizaje se basa en lo que sabemos llevado a nuevos contextos, dicha estrategia universal parece depender más de características individuales y probablemente del hecho de que habría que hacerla transparente en las explicaciones gramaticales y en la presentación de reglas. En una época caracterizada por las migraciones y 
LA ADQUISICION DEL SISTEMA VERBAL DEL ESPAŃOL. DATOS EMPÍRICOS DEL PROCESO DE APRENDIZAJE DEL ESPAŃOL COMO LENGUA EXTRANJERA.

Maria Cecilia Ainciburu (ed.). Frankfurt: Peter Lang, a. M., 20 i 7.

Graciela VÁzQuez

estudios que se llevan a cabo en diferentes países la importancia de la lengua materna tiende a minimizarse como origen de producciones idiosincrásicas. Este fenómeno resulta interesante en el contexto de la movilidad europea, tanto a nivel laboral como académico.

\section{Capítulo 5: Pel-wen Mao "Distinción aspectual para sino hablantes de ElE"}

Partiendo de su experiencia docente, la autora expone las dificultades de la alternancia imperfecto/pasado simple tanto para aprendientes como enseñantes sino hablantes. De las líneas de investigación existentes, opta por una perspectiva cognitiva de la influencia que podría ejercer la L1 (chino mandarín) y la interacción entre aspecto léxico y gramatical. Tras una descripción del aspecto en la L1 y una revisión de los estudios publicados sobre el tema, se presentan los resultados de un estudio empírico en el aula (actividades de reconocimiento) con hablantes monolingües que nunca han estado más de tres meses en un país hispanohablante. Las respuestas obtenidas a las preguntas sobre la influencia de la L1 y el aspecto léxico semántico no son concluyentes aunque se constata una tendencia a recurrir a la L1 como forma de reducir la carga cognitiva. En lo que respecta al aspecto léxico y semántico, se trata de categorías que influyen considerablemente aunque no de modo lineal. Esta constatación pareciera apoyar una verdad universal de la interlengua donde es difícil que se estabilicen las estructuras opacas si el sistema en general no detenta estabilidad. Dado que el chino mandarín no pertenece a la familia de lenguas romances, resulta interesante constatar cómo varía la interlengua en relación a la distancia tipológica y qué determina el proceso de fosilización. 


\section{Carítulo 6: Blanca Palacio Alegre "Oposición imperfecto-IndeFInidido y tipos de predicados:}

\section{UN ESTUDIO CON APRENDIENTES ALEMANES"}

El estudio realizado tiene como objetivo constatar con estudiantes universitarios germano parlantes de nivel A2 si existen diferencias significativas en cuanto al número de errores en la selección entre imperfecto e indefinido. El estudiantado fue expuesto a dos formas diferentes de instrucción: una explicación aspectual y otra temporal-aspectual aplicadas a cuatro categorías de predicados (estados, actividades, realizaciones y logros). Las actividades y la tarea (una y la misma para ambos grupos) fueron diseńadas según los planteamientos de la gramática cognitiva y el procesamiento del input. El comportamiento del tipo de predicado "logros" resulta ser la parte más productiva de la investigación. Teniendo en cuenta que se trata de comprobar la eficacia o no de diversas formas de instrucción, parece pertinente la limitación que se menciona: la autora no era quien impartía el curso. Hubiéramos apreciado más información acerca de la lengua instrumental utilizada, ya que se trata de un curso de principiantes, y al mismo tiempo en qué medida existía un grado de familiaridad con los instrumentos de medición y con el material didáctico.

\section{Capítulo 7: Patricia Rodríguez lópez "Análisis de interlengua de los rasgos gramaticales y}

\section{LÉXICO-SEMÁNTICOS CON LOS VERBOS SER Y ESTAR"}

El capítulo ofrece una extensa bibliografía anotada de la descripción gramatical de los verbos en cuestión tanto desde la perspectiva de la gramática normativa como pedagógica. Ofrece asimismo un resumen de los estudios de adquisición tanto en lengua materna como en E/LE. El grupo 
LA ADQUISICIÓN DEL SISTEMA VERBAL DEL ESPAŃOL. DATOS EMPÍRICOS DEL PROCESO DE APRENDIZAJE DEL ESPAŃOL COMO LENGUA EXTRANJERA.

Maria Cecilia Ainciburu (ed.). Frankfurt: Peter Lang, a. M., 20 i 7.

Graciela VÁzQuez

investigado es germano parlante y se estudian errores en la producción escrita en cinco niveles. El corpus se obtuvo en dos instituciones alemanas. La motivación de la autora es doble: la carencia de investigaciones en el mundo germano parlante y su experiencia docente. Sus preguntas de investigación son las siguientes: ¿Los errores de ser/estar guardan una correlación con el nivel de dominio de la lengua? ¿Existe una correlación entre tipo de error y estructura que los contiene? ¿Qué relación existe entre errores gramaticales y léxico-semánticos? El análisis llevado a cabo arroja resultados que difieren de las investigaciones realizadas con estudiantes anglófonos lo que parece sugerir que "ser/estar" plantea problemas de adquisición estrechamente ligados a su carácter de estructura opaca, y que, como muchos otros errores, resultan inseparables del nivel de proficiencia global. La transitoriedad y fosilización de algunos errores resulta instigadora teniendo en cuenta que la autora subraya la importancia de las implicaciones didácticas de su trabajo. Precisamente por la falta de estudios de esta naturaleza, se trata de un capítulo interesante que sugiereque la gramática pedagógica debería prestar más atención a lo que revelan los estudios de adquisición a la hora de explicar y presentar las reglas correspondientes.

\section{Capítulo 8: Carlos Soler Montes "La variación gramatical y el aprendizaje de los tiempos}

VERBALES DEL PASADO EN EL CONTEXTO DEL ESPAÑOL COMO LENGUA EXTRANJERA"

El objetivo que guía ese capítulo radica en la necesidad de incluir el fenómeno de la variación lingüística y, más importante aún, el de la variación gramatical, tanto en la formación del profesorado como en los materiales de enseñanza, tomando como ejemplo el pretérito compuesto 
perfecto y su tratamiento en los libros de texto. En primer lugar, el autor revisa los usos de los tiempos del pasado y proporciona una excelente descripción de los mismos para el castellano peninsular y de algunos países latinoamericanos. A continuación, reflexiona sobre su tratamiento en los libros y sugiere hacer uso de bancos de datos en línea para incluir datos con información como la que se ofrece en su investigación. Al final, analiza las explicaciones que se presentan en los materiales actuales. La segunda parte del capítulo está dedicada a un estudio empírico del pretérito perfecto compuesto utilizando el CAES (Corpus de Aprendices de Español) en seis grupos lingüísticos diversos y cinco niveles de proficiencia. Se trata, en concreto, de analizar la aparición del tiempo en cuestión, los valores y usos y la corrección morfológica otorgando especial atención a los rasgos perfectivo y pasado lejano, que coinciden en gran parte con los usos del mundo hispano. Los resultados demuestran que los libros simplifican en exceso los usos del tiempo. El alumnado utiliza el tiempo no necesariamente como lo determinan los planes curriculares y gramáticas en boga sino como lo harían personas nativas en situaciones reales de comunicación. Las consecuencias de dicha afirmación son fundamentales para las evaluaciones de carácter internacional que suelen poner en tela de juicio la posibilidad de conocer las diversas variantes del mundo hispano hable.

\section{Capítulo 9: Marco Antonio lópez "El uso del imperfecto e indefinido por parte de}

\section{eSTUDIANTES SUECOS DE ELE CON NIVEL A2,B1 y B2"}

Tras hacer una revisión de estudios relacionados con la selección de los tiempos del pasado, y dando cuenta de aquellos elementos que adopta para 
LA ADQUISICION DEL SISTEMA VERBAL DEL ESPAŃOL. DATOS EMPÍRICOS DEL PROCESO DE APRENDIZAJE DEL ESPAŃOL COMO LENGUA EXTRANJERA.

Maria Cecilia Ainciburu (ed.). Frankfurt: Peter Lang, a. M., 20 i 7.

Graciela VÁzQuez

su propia investigación cualitativo-cuantitativa, el autor de este capítulo describe primero el uso de los verbos de logro y estado y en segundo lugar focaliza la adquisición de los tiempos del pasado por parte de estudiantes suecos cuya lengua nativa, como en el caso del alemán y el chino, marca lo aspectual a través de partículas. La producción del imperfecto y del indefinido en dichos estudiantes y en un grupo de control de personas nativas es similar en el contexto de contar historias en el pasado. El uso del tiempo verbal y el aspecto léxico es aparente. La preocupación didáctica sobresale como un aspecto fundamental de la investigación.

\section{Capítulo 10: Marta Baralo Otonello "Significado psicolingüístico y didáctico de los} DATOS EMṔ́RICOS SOBRE LA ADQUIISICIÓN DEL SISTEMA VERBAL EN ESPAÑOL NO NATIVO".

En este último capítulo, Marta Baralo hace una evaluación de los trabajos presentados tanto desde la vertiente de la investigación básica como la aplicada, y destaca la contribución de los mismos al campo de la adquisición del sistema verbal no nativo, como así también a su didáctica. Asimismo, esta investigadora de larga trayectoria en el campo de la lingüística aplicada aprovecha los capítulos para reflexionar sobre las características de la interlengua y sobre los desafíos que plantean diversas técnicas de investigación a la hora de establecer la validez de los datos, refiriéndose en todo momento a ejemplos concretos de las investigaciones presentadas. La reflexión va más allá de los límites de la teoría y establece una relación directa con formas de instrucción gramatical explícitas y con las descripciones gramaticales, léxicas y semánticas. Cada capítulo ha hecho una contribución particular y concreta, aunque todavía estamos lejos de comprender en toda su magnitud 
los procesos de cognición en personas adultas no nativas, y de saber cómo implementar esos conocimientos en la práctica docente.

De la consulta y lectura atenta de este material se beneficiarán docentes de ELE a la hora de proporcionar explicaciones fundadas, e investigadoras e investigadores noveles para plantearse futuras investigaciones. En ese sentido, la bibliografía es un instrumento valioso para quienes quieran seguir investigando sobre el tema. Dichas investigaciones se beneficiarían si: - se dejara de presentar el imperfecto y pasado simple simultáneamente como si fueran comparables. No lo son: uno es tiempo, el otro modo.

- erradicáramos la ilusión de que el concepto de dificultad se basa en la "carencia" de elementos similares en la L1. Las lenguas pueden carecer de marcas pero no de funciones. Son los errores intralingüísticos los que demuestran las posibles limitaciones de las explicaciones gramaticales y las aparentes contradicciones del sistema.

- se analizara en más profundidad el fenómeno de la fosilización y las estructucturas potencialmente fosilizables.

- se abandonaran mitos como los que se expresan a través de informaciones del tipo "la selección del subjuntivo-indicativo es uno de los aspectos más complicados en ELE".

- se reflexionara más sobre el valor simbólico de la corrección en contextos escolares y universitarios, y en estos últimos se introdujeran las variedades del espańol como criterio normativo.

- se tuviera en cuenta qué relación guarda la aparición y no aparición de errores en relación al grado de dominio manifestado a través de los descriptores del Marco. 
CARACOL, SÃo PAULO, N. 16, JUL./DEZ. 2018

LA ADQUISICION DEL SISTEMA VERBAL DEL ESPAÑOL. DATOS EMPÍRICOS DEL PROCESO DE APRENDIZAJE DEL ESPAŃOL COMO LENGUA EXTRANJERA.

Maria Cecilia Ainciburu (ed.). Frankfurt: Peter Lang, a. M., 20 i7.

Graciela VÁzQuez

- se partiera de la base de que las formas idiosincrásicas no denotan simplemente estrategias y elementos adquiridos sino grados de dificultad entendida como operaciones cognitivas más amplias pero fallidas.

- se tuviera más en cuenta el peso de lo correcto frente a lo no correcto.

- se aceptara que en una lengua no aprendemos nada competa mente hasta haberlo aprendido casi todo. 